\title{
Uso de plataformas digitales para la enseñanza de la física en estudiantes universitarios
}

\section{Use of digital platforms for teaching physics to undergraduate students}

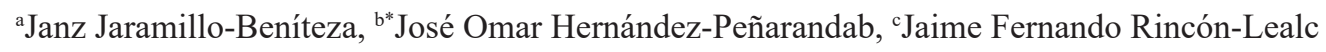 \\ ${ }^{a}$ Maestría en prácticas pedagógicas, j_jaramillo1@unisimon.edu.co, https://orcid.org/0000-0002-5988-2699, Institución Educativa \\ Juan Bautista La Salle, Cúcuta, Colombia \\ b*Docente, elpensador72@gmail.com, https://orcid.org/0000-0001-8017-3195, Institución educativa San Francisco de Sales, Cúcuta, \\ Colombia \\ 'Estudiante de Ingeniería Industrial, jaimefernandorlea@ufps.edu.co, https://orcid.org/0000-0003-4747-0360, Universidad Francisco \\ de Paula Santander, Cúcuta, Colombia
}

Forma de citar: Jaramillo-Benítez, J., Hernández-Peñaranda, J. O.., \& Rincón-Leal, J. F.. (2020). Uso de plataformas digitales para la enseñanza de la física en estudiantes universitarios. Eco Matemático, 11

(2), 81-86

Recibido: 6 Abril 2020

Aceptado: 21 Mayo 2020

\section{Palabras clave}

Movimiento armónico simple;

Aprendizaje;

Tic

\begin{abstract}
Resumen:El objetivo de la presente investigación es identificar la incidencia que tiene el uso de las plataformas digitales en el aprendizaje del Movimiento Armónico Simple en los estudiantes de I semestre del programa de Licenciatura en Matemáticas de la Universidad Francisco de Paula Santander en el primer semestre de 2021. El estudio es bajo el enfoque cuantitativo con un diseño cuasiexperimental de alcance descriptivo, con una muestra de 25 estudiantes, con 16 estudiantes de género femenino y 9 de género masculino, se utilizó un pre test y pos test de preguntas liberadas del ICFES aplicadas en Google Form para la recolección de la información el análisis se realizó con el software estadístico SPSS V 23, se determinó que el 100\% de los estudiantes se conectan a las clases con su celular inteligente, las plataformas más utilizadas por ellos es Google meet para las clases remotas, la plataforma de apoyo a la docencia las cuales son de uso institucional, también el conocimiento y uso del software GeoGebra y las plataformas, Educaplay, G suite de Google, Colombia aprende, Kahoot y Genially, y que las TIC inciden de manera positiva para alcanzar un mejor aprendizaje de la física.
\end{abstract}

*Autor para correspondencia:j_jaramillo1@unisimon.edu.co

Doi: https://doi.org/10.22463/17948231.3204 


\section{Keywords \\ Simple harmonic \\ motion; \\ Learning; \\ Tic}

\begin{abstract}
The objective of this research is to identify the incidence of the use of digital platforms in the learning of Simple Harmonic Motion in first semester students of the Bachelor's degree program in Mathematics at the Universidad Francisco de Paula Santander in the first semester of 2021. The study is under the quantitative approach with a quasi-experimental design of descriptive scope, with a sample of 25 students, with 16 students of female gender and 9 of male gender, a pre-test and post-test of ICFES released questions applied in Google Form was used for the collection of information the analysis was performed with the statistical software SPSS V 23, it was determined that $100 \%$ of the students connect to classes with their smartphones, the platforms most used by them are Google meet for remote classes, the teaching support platform which are of institutional use, also the knowledge and use of GeoGebra software and the platforms, Educaplay, Google G suite, Colombia aprende, Kahoot and Genially, and that ICT have a positive impact to achieve a better learning of physics.
\end{abstract}

\section{Introducción}

Los docentes de física en la actualidad en el ejercicio de su profesión para orientar sus temas en el aula de clase, deben implementar diferentes estrategias didácticas que posibiliten a los estudiantes aprender de manera interactiva , lo cual implica que el docente busque una serie de plataformas digitales y otros medios para crear ambientes de aprendizaje adecuados en donde las clases sean de forma dinámica teniendo en cuenta que la mayoría de los estudiantes universitarios son nativos digitales.

Del mismo modo, en la sociedad actual se encuentra inmersa en esta nueva normalidad y por ello se empezó a trabajar, a estudiar y realizar otras actividades desde la seguridad de sus hogares, para así no contagiarse y no contagiar a los demás, es por ello que en el campo de la educación comenzando desde la básica primaria hasta los posgrados universitarios que eran presenciales, se vieron en la obligación de buscar e implementar nuevas estrategias y metodologías, integrándolas al uso de herramientas tecnológicas para realizar el proceso de enseñanza y aprendizaje de manera remota.

Teniendo en cuenta el enfoque estratégico de las TIC del 2015 presentado para la educación en América Latina y el Caribe, para generar la actualización de las prácticas educativas al igual que los contenidos, los cuales deberían estar acordes a los avances de la nueva sociedad del conocimiento, en esta misma lógica, Colombia reafirma en el 2009 con la ley 1341 la implementación de las TIC, la cual define como: una reunión de tecnologías de la información y las comunicaciones para el beneficio de cualquier campo de acción, tanto así que existe un ministerio de las TIC el cual es el encargado y responsable de coordinar la articulación de las mismas en el plan de educación, fortaleciendo el ministerio de educación con el objetivo que en todas instituciones educativas de educación básica primaria, básica secundaria, media y universitaria gestione la implementación y el uso de aulas dotadas de herramientas y plataformas digitales para el proceso de enseñanza y aprendizaje (Rodríguez et al., 2017) y (Martinéz,2016).

Es por ello que el objetivo de la presente investigación es identificar la incidencia que tiene el uso de las plataformas digitales en el aprendizaje del Movimiento Armónico Simple (MAS) en los estudiantes de I semestre del programa de Licenciatura en Matemáticas de la Universidad Francisco de Paula Santander, en estos momentos de clases remotas mediadas por las TIC, y atendiendo a las necesidades de la educación actual. 
Es por ello que los docentes de física $\mathrm{y}$ de manera general vienen reestructurando sus prácticas pedagógicas a prácticas innovadoras dentro y fuera del aula, fomentando el uso de las plataformas digitales en el proceso de enseñanza y aprendizaje de los estudiantes en asignaturas de física I, construyendo y fortaleciendo las nuevas competencias del siglo XXI, para que sean utilizadas a lo largo de la vida académica y profesional (MEN, 2017).

Esta investigación se realiza con el fin de aportar una serie de plataformas digitales y el uso de dispositivos móviles a los estudiantes, para que así logren ser competentes dentro del mundo actual y así mismo puedan hacer uso de las plataformas tecnológicas que ya conocen, para la solución de problemas planteados y de la realidad (Ortiz \& Romero, 2015) y (Rincón Leal et al., 2019) desde el campo del estudio del Movimiento Armónico Simple el cual es un movimiento oscilatorio en el cual se desprecia la fricción y la fuerza de restitución es proporcional a la elongación. Al cuerpo que describe este movimiento se le conoce como oscilador armónico y su uso en un reloj de péndulo, amortiguador elastométrico, pistón en el motor de un automóvil, entre otras situaciones (Díaz Pinzón, 2018)

El uso de las plataformas digitales facilita una serie de alternativas que mejoran y facilitan el trabajo del estudiante y se deben utilizar como estrategia de los procesos de enseñanza - aprendizaje para el estudiante en temas como el movimiento armónico simple (Basuki, 2019), la implementación de las TIC en los contextos escolares pone en evidencia lo importante de una nueva definición de roles, especialmente, para los estudiantes y docentes universitarios, es por ello que se resalta que el uso de las plataformas digitales en los estudiantes generan una mayor autonomía y responsabilidad en el proceso de aprendizaje, lo que obliga al docente a salir de su rol clásico como única fuente de conocimiento y pasa a ser un guiador del aprendizaje es por ello que los profesores al momento de seleccionar la plataforma digital los diferentes estilos de aprendizaje (Jaramillo et al., 2020) puesto que esto es un factor determinante en el momento del general ambientes adecuados de aprendizaje de clases se física o de cualquier asignatura, a su vez, es importante resaltar que la tecnología posee algunas desventajas como: menor seguridad para la sociedad, manejo inadecuado, fácil adicción de quiénes lo utilizan, contaminación ambiental, y la suplantación en el momento de evaluar (Rodríguez Contreras et al., 2017).

Por lo tanto, para la nueva modalidad virtual en educación, el educador no sólo debe ser capaz de aplicar las plataformas digitales al servicio de la educación, sino también, debe tener las habilidades al igual que destrezas para diseñar nuevos escenarios educativos donde los estudiantes puedan aprender a moverse y poder participar en el espacio telemático (Echeverria,2000), para (Barráez et al., 2020)la integración de las TIC en la educación ha abierto grandes posibilidades para enriquecer los procesos de enseñanza y de aprendizaje en los espacios virtuales en los campos universitarios.

\section{Metodología}

La presente investigación se encuentra en el enfoque cuantitativo bajo los descrito por(Hernández et al., 2014), con un diseño no experimental de alcance descriptivo (Spiegel, 2000), el estudio se realizó en cuatro fases:

Fase 1. Aplicación del pretest en el cuestionario de google form, una calificación cuantitativa de los conocimientos del Movimiento Armónico Simple con preguntas liberadas del cuadernillo de las pruebas saber 11 (2014 a 2018)

Fase 2. Implementación de las plataformas digitales: Gogebra, Quizziz, G suite Google, Kahoot, Genially, Emaze y Colombia aprende para las clases de física en el tema de movimiento armónico simple. 
Fase 3. Aplicación del pos test en el cuestionario de google form, una calificación cuantitativa de los conocimientos del Movimiento Armónico Simple después de la implementación de las plataformas digitales.

Fase 4. Análisis de resultados y conclusiones de los estudiantes en las asignaturas de Física I, del programa académico de Licenciatura en Matemáticas de la Universidad Francisco de Paula Santander.

\section{Resultados}

El total de la población estuvo conformada por 320 estudiantes entre 17 y 30 años de edad que actualmente están inscritos en el programa académico de licenciatura en matemáticas de la universidad Francisco de Paula Santander, Colombia. Con una muestra de 25 alumnos pertenecientes al I semestre, 16 estudiantes equivalente a $56 \%$ de género femenino y 9 alumnos equivalente a $44 \%$ de género masculino, de los cuales el $40 \%$ son de estrato 1 y $60 \%$ de estrato 2, el análisis de información evidencia que el 20\% de los estudiantes tienen computador de escritorio, el $62 \%$ computador portátil, y el $100 \%$ teléfono inteligente, dispositivos por los cuáles se conectan a las clases por la plataforma de Google Meet, puesto que la universidad brinda a los docentes (Google Workspace for Education) para la conexión de las clases sincrónicas, y además la universidad cuenta con una plataforma Moodle denominada Plataforma de Apoyo a la Docencia con sus siglas PLAD, la cual es una plataforma web 2.0 que permite la interacción entre el estudiante y el docente de todas las asignaturas.

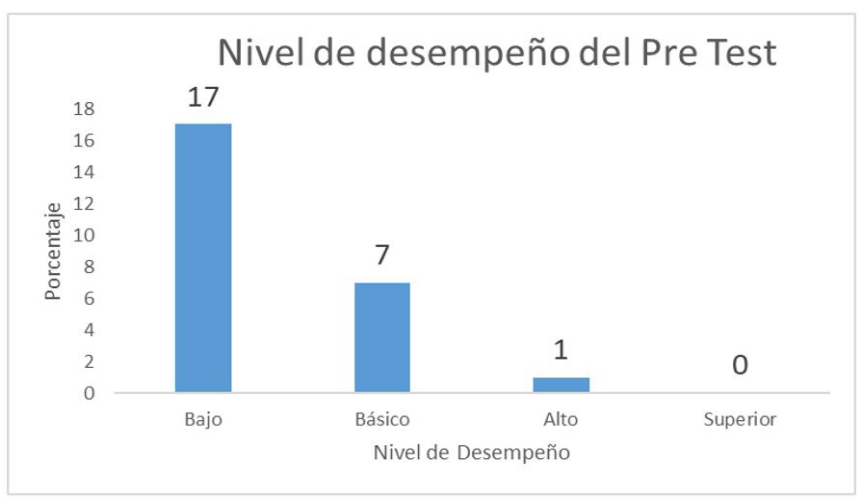

Figura 1. Nivel de desempeño del pre test

La figura 1 evidencia el promedio de cada uno de los estudiantes de I semestre de programa de licenciatura en Matemáticas el cual tiene un promedio general de 2,6 quedando 17 estudiantes equivalente a $68 \%$ en nivel bajo, 7 estudiantes equivalente a $28 \%$ en nivel básico, 4 estudiantes equivalente a $4 \%$ en nivel alto y 0 en nivel superior.

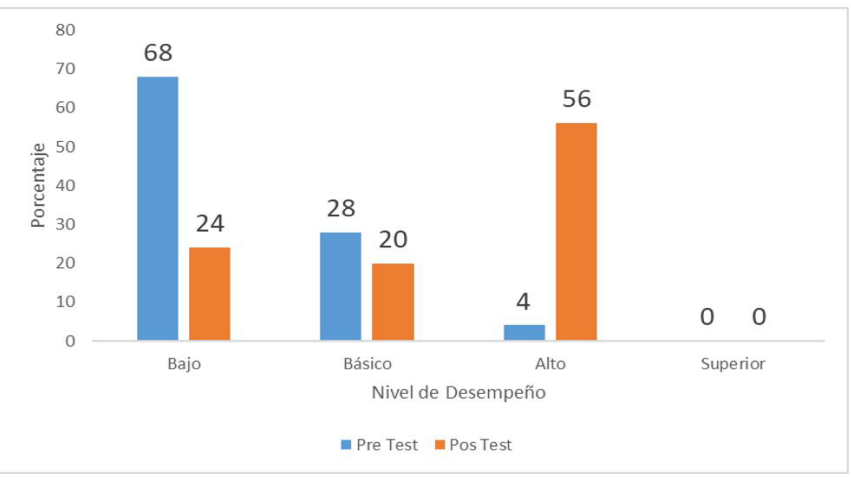

Figura 2. Comparación del Nivel de Desempeño en el Pre Test y Pos Test

La figura 2 evidencia que el promedio general en el pos test fue de 3,58 en comparación con el promedio del pre test de 2,6 se obtuvo una ascenso a nivel general en la media de 0,96 en cual es una diferencia significativa, del mismo modo el post tes en el nivel de desempeño bajo, descendió de $68 \%$ a $24 \%$ es decir $44 \%$ equivalente a 11 estudiantes, en el nivel de desempeño básico bajo de $28 \%$ a $20 \%$ es decir $8 \%$ equivalente a 2 estudiantes, en el nivel de desempeño alto ascendió de $4 \%$ a $56 \%$ es decir subió $52 \%$ equivalente a 13 estudiantes, siendo así estas diferencias positivas y poder afirmar que el uso de 
las plataformas digitales incide de manera positiva en el aprendizaje del movimiento armónico simple en los estudiantes de I semestre de licenciatura en Matemáticas de la universidad Francisco de Paula Santander.

\section{Conclusiones}

Como conclusión se puede afirmar que en el I semestre del programa académico de licenciatura en Matemáticas existentes más estudiantes de género femenino que masculino y el estrato académico más alto es el 2, el cual evidencia que los estudiantes presentan vulnerabilidad en sus condiciones socioeconómicas, sin embargo, en estos momentos se idearon para recibir clases de manera remota desde sus hogares.

De la misma manera el uso adecuado e interactivo de las plataformas digitales incide de manera positiva para el aprendizaje del movimiento armónico simple, al igual que otros temas en cualquier asignatura, ya que en la presente investigación se obtuvo una diferencia significativa en los promedios generales del pre test y pos test.

\section{Referencias}

Barráez, D. P., Sánchez-palacios, L., Miranda, A. J., Motenegro, S. L., Militar, A., Vaillant-delis, M., \& Bonaerenses, M. (2020). Personas con Discapacidad y Aprendizaje Virtual: Retos para las TIC en Tiempos de People with Disabilities and Virtual Learning: Challenges for ICT in the Days of Covid-. Revista Internacional Tecnológica-Educativa Docentes 2.0, 8(26650266), 204-211. https://ojs.docentes 20. com/index.php/revista-docentes $20 /$ article/ view/91/232

Basuki, K. (2019). Sears y Zemansky, Fisica universitaria volumen 1 13o Edición. In Enerojunio 2019 (Vol. 53, Issue 9). www.journal. uta45jakarta.ac.id
Díaz Pinzón, J. E. (2018). Aprendizaje de las Matemáticas con el uso de Simulación. Sophia, 14(1), 22-30. https://doi.org/10.18634/ sophiaj.14v.1i.519

Echeverría, J. (2000). Educación y tecnologías telemáticas. Revista Iberoamericana De Educación, 24, 17-36. https://doi.org/10.35362/ rie240995

Hernández, R., Fernández, C., \& Baptista, P. (2014). Diferencias entre los enfoques cuantitativo y cualitativo. Metodología de La Investigación, 12.

http://euaem 1 . ua em.mx/bitstrea m/ $\mathrm{h}$ and le/123456789/2774/506_1. pdf? sequence $=1 \&$ is Allowed $=y$

Jaramillo, J. E., Rincon Leal, J. F., \& Rincon Leal, O. L. (2020). Impact of learning styles on multiple intelligences in first semester math students. Journal of Physics: Conference Series, 1645(1). https://doi.org/10.1088/17426596/1645/1/012015

Martínez, D. M. (2016). Un acercamiento a la comprensión del uso de TIC en la educación básica y media en Colombia. Recuperado de: http://hdl.handle.net/20.500.12209/532

MEN. (2017). Plan Nacional de Educación 2016 -2026 El camino hacia la equidad. In Plan Nacional de Educación 2016 -2026

Ortiz, L., \& Romero, M. (2015). La implementación de las TIC en el aula de matemáticas: Una mirada sobre su concepción en el siglo XXI. Universidad Pedagógica Nacional., 9, 27

Rincón Leal, L., Vergel Ortega, M., \& Paz Montes, L. S. (2019). Mobile devices for the development of critical thinking in the learning of differential equations. Journal of Physics: Conference Series, 1408(1). https://doi.org/10.1088/1742- 
$6596 / 1408 / 1 / 012015$

Rodríguez Contreras, J. L., Romero Pabón, J. C., \& Vergara Ríos, G. M. (2017). Importancia De Las Tic En Enseñanza De Las Matemáticas Importance of Ict in Teaching Mathematics. Revista MATVA, 2, 41-49

Spiegel, M. (2000). Estadística (2nd ed.). Mc Graw Hill 